\title{
Oral Abstract Presentations at the 8th Canadian Conference on Dementia (CCD), Ottawa, October 2015
}

http://dx.doi.org/10.5770/cgj.18.217

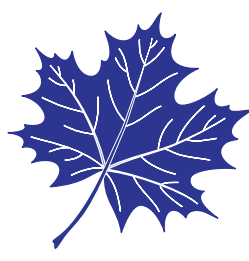

ORAL ABSTRACTS

Vascular Risk Factors and Subcortical Arteriosclerotic Leukoencephalopathy, But Not Alzheimer Lesion Load, Are Associated with Development of Psychosis in Alzheimer's Disease

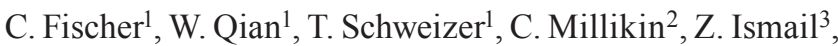
E. Smith $^{3}$, L. Lix ${ }^{4}$, P. Shelton ${ }^{4}$, D. Munoz ${ }^{1}{ }^{1}$ St. Michael's Hospital; ${ }^{2}$ University of Manitoba; ${ }^{3}$ University of Calgary; ${ }^{4}$ University of Manitoba.

Background/Objectives: Psychosis (delusions or hallucinations) is a common clinical feature in patients with Alzheimer's disease (AD), and is estimated to affect up to half of this patient population. Psychosis is associated with increased cognitive and functional decline as well as greater caregiver burden. However, lack of understanding of the pathobiological mechanisms behind psychosis limits our ability to manage and treat these symptoms. The current literature is mixed, with some studies showing a correlation between psychosis and Alzheimer pathology, while others find no such association. Using data from the large National Alzheimer's Coordinating Center (NACC) database, we aimed to determine the demographic, clinical, and neuropathological features associated with psychotic symptoms, separated into delusions and/or hallucinations, in patients who were clinically or neuropathologically diagnosed with AD.

Methods: All data were obtained from the NACC database. Our sample of consisted of 1073 subjects broken down into: 1) 890 clinically diagnosed AD (cAD) patients with neuropathology data, and 2) 728 neuropathologically confirmed AD (npAD) patients based on the Consortium to Establish a Registry for Alzheimer's Disease (CERAD) criteria of "definite" AD with any clinical diagnosis. The two groups overlapped, but each was reviewed separately. Delusions and hallucinations were identified by the delusional and hallucinatory sub-scores of the Neuropsychiatric Inventory (NPI-Q), respectively.

Results: Over one-third of our AD subjects (cAD and npAD) had psychotic symptom(s) during the course of their illness.
There were no apparent differences between patients with psychosis (cAD +P n=307; npAD $+\mathrm{P} n=271$ ) and without psychosis (cAD-P n=583; npAD-P $n=457$ ), defined globally, on functional (FAQ) and cognitive outcomes (MMSE, global CDR). However, further breakdown by psychotic symptoms showed that patients with hallucinations $(\mathrm{cAD}+\mathrm{H}$ $\mathrm{n}=57$; $\mathrm{npAD}+\mathrm{H} \mathrm{n}=52$ ) were more severely cognitively and functionally impaired at the last visit prior to death, while patients with delusions (cAD $+D n=164$; npAD $+D n=140$ ) were less impaired than non-psychotic patients, consistent across both cAD and npAD groups. We found that, in the cAD group, psychosis was associated with greater levels of plaques and tangles, but this association was not present in the npAD group. Lewy body pathology, sub-cortical arteriosclerotic leukoencephalopathy (SAL) and vascular risk factors, particularly a history of hypertension and diabetes, were associated with psychotic symptoms in both the cAD and npAD groups.

Conclusion: Markers of AD pathology (plaques, tangles) are associated with psychotic symptoms in clinically diagnosed AD patients. However, in neuropathologically confirmed AD patients, AD pathology does not correlate with psychotic symptoms, suggesting that the association in the clinical group is driven by misdiagnosis. Instead, in the neuropathologically confirmed AD group, psychosis is associated with Lewy body pathology, vascular risk factors, and vascular pathology. The findings suggest that modifiable factors such as vascular pathology may play a role in psychosis in AD.

Predicting Alzheimer's Disease Development: a Comparison of Cognitive Criteria and Associated Neuroimaging Biomarkers

B. Callahan, J. Ramirez, C. Berezuk, S. Duchesne, S. Black. Sunnybrook Health Sciences Centre, Toronto, Canada.

Background: There exist multiple criteria for identifying mild cognitive impairment (MCI) (Albert et al., 2011; APA, 
2013; Petersen, 2004). All require the presence of "objective impairment" in $\geq 1$ cognitive domains, typically including memory. These criteria have been crucial in conceptualizing MCI. However, to improve their ability to assess dementia risk, four critical issues remain. First, it is unclear which cut-off on normative tests should be used to operationalize cognitive impairment. Second, no criteria specify how many measures of a cognitive domain should be administered. Third, it is unclear which domain(s) should be assessed in addition to memory, if any, to determine dementia risk. Fourth, although current guidelines (Albert et al., 2011) emphasize the importance of considering biomarkers, it is unclear how these inform dementia risk above and beyond cognitive testing. This study aims to determine whether prediction of dementia is improved by: 1) using different cut-off scores on standardized tests to define cognitive impairment; 2) assessing memory using one or two tests; 3 ) considering non-memory domains; and 4) accounting for genetic and image-based anatomical biomarkers.

Methods: Non-demented seniors from the Alzheimer's Disease Neuroimaging Initiative $(\mathrm{N}=494)$ underwent cognitive assessment for global function (Mini-Mental State Exam [MMSE]), memory (Logical Memory Story A Delayed Recall [LM-II], Rey Auditory Verbal Learning Test Delayed Recall [AVLT]), language (Category Fluency, Boston Naming Test), and executive functions (Trails A \& B). Standardized scores were calculated based on ageadjusted norms. Biomarkers included brain parenchymal fraction (BPF), ventricular cerebrospinal fluid, hippocampal (HP) and white matter hyperintensity volumes, obtained via automated processing, and APOE $\varepsilon 4$ status. The presence of $\mathrm{AD}$ at 24 months was defined as: 1) MMSE <26; 2) Clinical Dementia Rating Scale $\geq 0.5$; and 3) positive NINCDS/ ADRDA criteria for probable AD. Six binary variables were created based on scores $\leq-1,-1.5$ or -2 standard deviations (SD) on one (LM-II or AVLT) or two (LM-II and AVLT) memory tests. The accuracy of these cut-offs to predict dementia was tested using the area under the curve (AUC) for receiver operating characteristic analysis. Cut-offs with AUC $>0.75$ were entered into logistic regression analyses, with age, sex, education, and MMSE in a first block. The second and third blocks included non-memory tests and biomarkers, respectively.

Results: The most accurate cut-off for predicting dementia at 24 -months was $\leq-1 \mathrm{SD}$ on two memory tests $(76.92 \%$ accuracy; $\mathrm{AUC}=.788$ ). In addition to this cut-off, dementia was associated with the presence of two APOE 84 -positive alleles ( $\mathrm{B}=1.15, \mathrm{SE}=0.45, p=.011)$. The second most accurate cut-off was $\leq-1.5 \mathrm{SD}$ on one test $(68.02 \%$ accuracy; $\mathrm{AUC}=.765)$. In addition to this cut-off, dementia was associated with HP volume $(\mathrm{B}=-4.84, \mathrm{SE}=2.43, p=.047), \mathrm{BPF}(\mathrm{B}=-15.97, \mathrm{SE}=7.58$, $p=.035)$, and presence of one $(\mathrm{B}=0.60, \mathrm{SE}=0.29, p=.042)$ or two APOE $\varepsilon 4$-positive alleles $(\mathrm{B}=1.08, \mathrm{SE}=0.45, p=.017)$.
Conclusions: Prediction of dementia is improved by defining cognitive impairment as performance $\leq-1$ SD on two memory tests. Clinicians or researchers administering one test should opt for a more stringent cut-off $(\leq-1 \mathrm{SD})$ and analyze available imaging data, particularly whole-brain and hippocampal volumes. When feasible, ascertaining APOE 44 status can further improve prediction.

\section{Atypical Antipsychotics for the Behavioural and Psychological Symptoms of Dementia in the Elderly: a Systematic Review and Network Meta-Analysis}

A. Johnston ${ }^{1}$, S. Kelly ${ }^{1}$, A. Bai ${ }^{1}$, L. Chen ${ }^{1}$, G. Wells ${ }^{1,2}$, A. Kotb $^{1}$, B. Skidmore ${ }^{3}$, T. Gomes ${ }^{4} .{ }^{1}$ Cardiovascular Research Methods Centre, University of Ottawa Heart Institute; ${ }^{2}$ School of Epidemiology, Public Health and Preventative Medicine, University of Ottawa; ${ }^{3}$ Consultant; ${ }^{4} \mathrm{Li} \mathrm{Ka}$ Shing Knowledge Institute of St. Michael's Hospital.

Background: Dementia is characterized by a progressive decline in cognitive ability and memory resulting in an impaired ability to function independently. Behavioural and psychological symptoms of dementia (BPSD) (e.g., psychosis, agitation/aggression) are common and constitute a major component of all dementia sub-types. These symptoms are often distressful to caregivers, and are a leading cause of long-term-care placement. A variety of drugs are used on and off-label for adults with BPSD who are unresponsive to behavioural or caregiver support. Indeed, choosing the "right" medication involves complex treatment decisions. Atypical antipsychotics (AAPs) are commonly used to manage symptoms of dementia; however, their use is controversial, given their limited benefits as demonstrated in placebo trials, along with a high risk of treatment emergent adverse events - particularly among elderly adults.

Objective: To identify, synthesize, and appraise the available randomized comparative evidence on the efficacy and safety of AAPs among community and long-term care-dwelling elderly patients with BPSD.

Methods: A systematic review and Bayesian network metaanalysis. Randomized controlled trials (RCT) were included if they had at least 10 participants (mean age $\geq 65$ years) diagnosed with dementia who were experiencing BPSD at baseline. Patients could be treated with any dose of 9 eligible AAP medications. Studies that compared an AAP to placebo, another AAP, or any active comparator were considered. To build the evidence base, we identified two high quality evidence syntheses that met our inclusion requirements. Two reviewers then screened the full text of their included studies for eligibility. To augment this evidence, a systematic literature search strategy was carried out in MEDLINE, 
EMBASE, PsychInfo, and the Cochrane Central Register of Controlled Trials (January 2009 to October 2014), along with a search for grey literature. Two reviewers screened titles/abstracts, reviewed full-text, extracted data, and carried out a critical appraisal using the Cochrane Risk of Bias tool. All eligible studies were included; however, data were only extracted from studies reporting outcomes of interest (5 efficacy, 4 safety).

Results: We included 37 unique RCTs published between 1995 and 2014, with 32 reporting outcomes of interest. On average, caregiver burden was reduced in patients who took olanzapine, risperidone, or quetiapine compared to placebo; however, the reductions were modest and not statistically significant. Similar results were found among patients living in long-term-care facilities. There were no significant differences between the AAPs and placebo, or among the AAPs, in reducing overall BPSD or individual symptom scores. We found a significantly higher risk of all-cause mortality associated with the use of any AAP relative to placebo (OR 1.9, 95\% Crl 1.19, 3.16). There were, however, no significant differences in mortality between individual AAPs. Sensitivity analyses revealed similar results for community and long-term-care dwellers. Many studies (43\%) had small sample size $(<100$ participants) and the quality of the reporting of trials varied.

Conclusions: Evidence suggests a modest benefit from the use of AAPs among elderly patients with BPSD. Given the increased risk of death, clinical judgment is required when applying these research findings to every day practice.

\section{Prevalence and Incidence of Dementia Among Indigenous Populations: a Systematic Review}

L. Warren ${ }^{1}$, S. Qiyun ${ }^{2}$, K. Young ${ }^{3}$, A. Borenstein ${ }^{4}$, A. Martiniuk ${ }^{5}{ }^{1}$ Dalla Lana School of Public Health, University of Toronto; ${ }^{2}$ Health and Rehabilitation Sciences, Western University; ${ }^{3}$ School of Public Health, University of Alberta; ${ }^{4}$ Department of Epidemiology and Biostatistics, College of Public Health, University of South Florida; ${ }^{5}$ George Institute for Global Health, Sydney, NSW, Australia.

Background: Indigenous populations may be at increased risk, compared with majority populations, for the development of dementia due to lower education levels and socioeconomic status, higher rates of diabetes, hypertension, cardiovascular disease and alcohol abuse, an aging population structure, and poorer overall health. This is the first systematic review investigating the prevalence and incidence of dementia in Indigenous populations worldwide.

Methods: This systematic review was conducted in accordance with PRISMA guidelines. We searched MEDLINE, Embase,
PsycInfo, and grey literature for relevant articles published up to April 2015. Studies were included if they reported prevalence or incidence, the disease typically occurred after the age of 45 , the study population included Indigenous people, and the study was conducted in the general population. Prevalence rates for individuals $\geq 65$ were age-standardized to the 2014 Canadian population, where data permitted.

Results: Fifteen studies representing five countries (Canada, Australia, United States, Guam, Brazil) met the inclusion criteria. Four studies presented incidence data, which were all conducted in Guam, and 11 studies reported prevalence data. Seven of the prevalence studies were based on primary data collection and developed or modified cognitive assessment tools for use in indigenous populations, while the other four were based on pre-existing data. Dementia prevalence ranged from $0.5 \%$ to $20 \%$. The age-standardized prevalence rates were the same for the two Canadian studies (3.5\%), which was much lower than the age-standardized rates for Guam (16\%) and Australia (15-27\%). In Australia, the agestandardized prevalence rate was higher for the prevalence study $(27 \%)$ than it was for the study based on pre-existing data on treated dementia patients $(15 \%)$. Retrospective studies relying on medical records for diagnoses had much lower prevalence rates and a higher risk of bias than populationbased prospective studies performing their own diagnoses with culturally appropriate cognitive assessment methods.

Conclusion: The prevalence of dementia among Indigenous populations appears to be higher than it is for non-Indigenous populations. Despite a building body of evidence supporting the need for dementia research among Indigenous populations, there is a paucity of epidemiological research, none of which is of high quality.

Apolipoprotein E 84: Associations with Hippocampal Volumetrics and Verbal Episodic Memory Performance Across Alzheimer's Disease, Dementia with Lewy Bodies and Mixed Dementias

U. Saeed ${ }^{1,2}$, Q. Yu ${ }^{1}$, B. McIntosh ${ }^{1,3}$, N. Herrmann ${ }^{1,4}$, J. Ramirez ${ }^{1}$, E. Rogaeva ${ }^{5}$, S. Black ${ }^{1,6}$, M. Masellis ${ }^{1,7}$. ${ }^{1}$ Sunnybrook Health Sciences Centre; ${ }^{2}$ Institute of Medical Science, Faculty of Medicine, University of Toronto; ${ }^{3}$ Department of Medical Biophysics, University of Toronto; ${ }^{4}$ Department of Psychiatry, Faculty of Medicine, University of Toronto; ${ }^{5}$ Tanz Centre for Research in Neurodegenerative Diseases, Faculty of Medicine, University of Toronto; ${ }^{6}$ Brill Chair in Neurology, Department of Medicine, University of Toronto; ${ }^{7}$ Department of Neurology, Faculty of Medicine, University of Toronto.

Background: Alzheimer's disease (AD) and dementia with Lewy bodies (DLB) are the most common neurodegenerative 
dementias. There is an urgent need to develop disease-modifying agents to prevent, delay or slow the progression of these dementias at early stages. The development of such interventions is challenged by substantial clinical heterogeneity observed within a single clinical diagnosis and this may in part be due to mixed pathologies on a shared genetic background. For example, carriers of the Apolipoprotein E (APOE) gene $\varepsilon 4$ variant are known to have a higher risk of developing pathologically-proven $\mathrm{AD}$, $\mathrm{DLB}$, and mixed AD/DLB. In existing literature, the $\varepsilon 4$ allele is consistently associated with smaller hippocampal volumes among $\mathrm{AD}$ patients. To our knowledge, however, this association has not been studied in DLB and mixed dementias. This warrants further investigation to see if $\varepsilon 4$ consistently associates with hippocampal measures across different groups of dementias.

Objectives: 1) To investigate whether APOE $\varepsilon 4$ allele associates with hippocampal volumetric measures across dementia sub-types in a dose-dependent manner. 2) To investigate differences in hippocampal volumetrics across dementia subtypes based on an extreme genetic subgroup approach (i.e., non-carriers vs. homozygous carriers of $\varepsilon 4$ ). 3) To investigate the association between hippocampal volumes and verbal episodic memory within APOE sub-groups.

Methods: The study included 261 patients with a clinical diagnosis of dementia (160 AD, $46 \mathrm{AD}$ with small vessel disease [SVD], 35 mixed AD/DLB, 20 pure DLB). MRI data were pre-processed using the Semi-Automated Brain Region Extraction (SABRE) pipeline followed by estimation of hippocampal volumetrics using Sunnybrook Hippocampal Volumetry tools. The first analysis (objective 1) assessed dose-dependent effects of $\varepsilon 4$ on hippocampal volumetric measures by stratifying the sample into non-carriers "-/.", heterozygous carriers " $+/-$ ", or homozygous carriers " $+/+$ ". The second analysis (objective 2) examined hippocampal volumetric measures in the extreme subgroups of $\varepsilon 4$ " $+/+$ " vs. "-/-". The third analysis (objective 3 ) evaluated the linear relationship between hippocampal measures and performance on the California Verbal Learning Test (CVLT). Between-group differences in baseline hippocampal volumes were analyzed using analysis of covariance (ANCOVA). Linear regressions were used to examine the associations of hippocampal measures with CVLT.

Results: There were 104 APOE $\varepsilon 4$ "non-carriers" and 157 "carriers" in our sample (104 "-/-", 119 "'+/-", and 38 "'+/+"). There was a trend for total bilateral hippocampal volume (HV) and individual left and right $\mathrm{HVs}$ to be smaller in a dose-dependent fashion: $\varepsilon 4$ " $+/+$ " $<\varepsilon 4$ " $+/ /-"<\varepsilon 4$ " $-/ /-"$. The total bilateral $\mathrm{HV}$, and individual left and right $\mathrm{HVs}$ were significantly smaller $(p<.05$ for all) in " $+/+$ " " vs. " $-/$. " extreme $\varepsilon 4$ sub-groups. In $\varepsilon 4$ carriers, regression analysis revealed stronger associations of HVs with California Verbal Learning Test (List-A total recall, short delay cued recall, long delay free and cued recall, $p<.01$ for all) relative to non-carriers.

Conclusions: The results suggest that APOE $\varepsilon 4$ predicts greater hippocampal atrophy and episodic memory impairments across a sample of heterogeneous dementia patients. This hippocampal-memory association may serve as a reliable endophenotype of APOE $\varepsilon 4$ allele across dementia patients. 